\title{
Salvage Living Donor Liver Transplantation (SLDLT) as a Treatment Modality for Recurrent Hepatocellular Carcinoma (HCC)
}

\author{
Ahmed A. Abdelshafy, MD; M. Rady, MD; Waleed Ibrahim, MD \\ Department of General Surgery, Faculty of Medicine, Ain Shams University, Egypt
}

Background: Hepatocellular carcinoma (HCC) is the fifth most common cancer all over the world. Salvage living donor liver transplantation (SLDLT) has been proposed for patients who developed HCC recurrence after primary resection or liver deterioration in patients undergoing liver resection (LR).

Aim of the work: The aim of this study is to compare and evaluate the patient survival and cancer recurrence rates between patients who underwent (SLDLT) and (PLT) in our centers between 2014 and 2018.

Patients and methods: A retrospective study was conducted on total of $110 \mathrm{HCC}$ patients who underwent LDLT for HCC from January 2014 till January 2018 by the same surgical team at 3 centers (Ain Shams University Specialized Hospital, Wadi-EINile Hospital and Egypt Air Hospital). Patients were divided into two groups. Group A included primary LDLT for 95 cases which were within Milan criteria and group B for SLDLT was performed in 15 cases.

Results: Among the 110 HCC patients, 95 (86.4\%) underwent PLT, and 15 (13.6\%) underwent SLT for HCC recurrence after the primary resection. The operative time was significantly more ( $P$ value $=0.001)$ in the SLT recipients group, as the median operative time was (10) hours while it was (8) hours in the PLT recipients group. For all post-operative complications, no statistical significant difference was found between the PLT and SLT recipients. Similar rates were observed as regard the overall survival between the SLT and PLT recipients. However, the 1 year, 3 year and 5 year disease-free survival rates were $94.5 \%, 93.0 \%$, and $92.1 \%$ in PLT recipients, compared to recurrence that occurred in 1 patient after 9 months (6.7 \%) in SLT recipients which was not significantly different statistically.

Conclusion: Our study shows that SLT is a safe and technically feasible procedure for patients who developed HCC recurrence following primary liver resection. Also, the survival rates were similar to PLT.

Key words: Hepatocellular carcinoma, salvage living donor liver transplantation, liver resection.

\section{Introduction}

Hepatocellular carcinoma (HCC) is the fifth most common cancer all over the world. ${ }^{1}$ HCC now constitutes the most frequent cause of death in patients with cirrhosis. ${ }^{2}$

Liver resection (LR) and radio-frequency (RF) ablation are important curative lines for treatment of HCC in patients with child A cirrhosis with good liver reserve and function. ${ }^{3}$ Despite improved outcome and survival with those modalities, the most disappointing is the high 5- year tumor recurrence rates reaching $50 \%$ in LR and up to $80 \%$ in RF ablation. ${ }^{4}$

Primary liver transplantation (PLT) is surely the treatment of choice for such HCC patient as it also removes the whole liver with its precancerous potential. ${ }^{5}$ However PLT is hindered by organ shortage ${ }^{6}$ especially in Egypt due to absence of cadaveric program, long waiting list, waiting list drop-off and post-transplant life-time need for immune suppression.

Salvage living donor liver transplantation (SLDLT) has been proposed for patients who developed HCC recurrence after primary resection or liver deterioration in patients who underwent LR. ${ }^{7}$

In our retrospective study we compared and evaluated the patient survival and cancer recurrence rates between patients who underwent (SLDLT) and (PLT) in our centers between 2014 and 2018.

\section{Patients and methods}

A retrospective study was conducted on total of 110 HCC patients who underwent LDLT for HCC from January 2014 till January 2018 by the same surgical team at 3 centers (Ain Shams University Specialized Hospital, Wadi-EINile Hospital and Egypt 
Air Hospital).

Patients were divided into two groups. Group A included primary LDLT for 95 cases which were within Milan criteria and group B for SLDLT performed in 15 cases. The indication for SLDLT was either HCC recurrence after liver resection $(n=9)$ or progression to end stage liver disease $(n=6)$. Major liver resection was done in 4 cases while minor liver resection (less than 3 segments) was done in 11 cases. All recurrences for patients who underwent SLDLT were within Milan criteria.

All patients of PLT and SLT groups were compared regarding their age, gender, tumors number, largest tumor diameter, pre-operative level of AFP, post transplantation macro vascular invasion, and time of follow up.

A comparison of the operative characteristics was done in terms of operative time, loss of blood, postoperative Intensive Care Unit (ICU) stay, and overall post-operative hospital stay time.

Also we compared all the possible major postoperative complications between the two groups, as biliary complications, bleeding, postoperative intra-abdominal collection or abscess, and renal failure.

The over-all and disease-free survival rates of 1 year and 3 year were also compared between the SLT and PLT patients.

\section{Surgical technique}

All patients received a right lobe graft with a minimum Graft Weight Recipient Ratio (GRWR)>= (0.8). Recipient hepatectomy in both groups was conducted by "piggy back" technique preserving inferior vena cava.

But in the SLDLT group meticulous dissection of all adhesions at the cut surface of previous hepatectomy was of paramount importance to restore the normal anatomy before any dissections to avoid unnecessary bleeding and therefore minimizing intra operative blood loss.

Unlike the cadaveric graft hepatectomy, hilar dissection started at the "hilar plate" level aiming to obtain as long as possible of the hilar structures.

Left approach to dissect and separate the left hepatic artery (LHA) with its branches then segment 4 hepatic artery (MHA), if present, was preferred to avoid excessive traction during liver mobilization.

Following this step, dissection of the right and left hepatic bile ducts was done distal to the bifurcation, then disjoined. In the meantime, the blood supply of the main bile duct was carefully preserved.

The hepatoduodenal dissection was completed by dissecting and fully simplifying the portal vein. Afterwards, removal of the caudate lobe was done by ligating and separating all short hepatic veins and the hepatocaval ligament.

The left lobe was mobilized with dissection of the left hepatic vein (LHV) up to the MHV junction, after suspending the right hepatic vein. Lastly, when the donor's liver was removed and prepared, hepatectomy was completed by separating the hepatic veins and the portal vein, which was done as the last step to avoid congestion of the bowel and portal circulation.

As far as possible, total IVC clamping was avoided while completing the hepatic vein anastomosis to maintain the hemodynamic stability and renal functions. However, total IVC clamping was superior to partial clamping while venous reconstruction was done to ensure adequate venous drainage, in case the recipient was able to tolerate it.

The first step in right lobe transplantation was the RHV anastomosis, which was first prepared in order to create a large triangular ostium to ensure good outflow drainage. If there were any accessory hepatic veins they were anastomosed to the IVC which was done end to side. The MHV and LHV orifices were also closed. However, in some cases with accessory V8, it was anastomosed by synthetic graft to MHV orifice after preparing it.

The next step was the portal vein anastomosis which was done very carefully to obtain good inflow to the graft, so preparing of the recipient portal was done with adjustment of its length to avoid any kink that may happen after the end to end anastomosis. Following the portal vein anastomosis, portal clamping was released to start the graft reperfusion, also the hepatic vein clamp was removed where hemostasis was done and ensured. Satisfactory graft reperfusion was clinically justified by certain signs intraoperative as the homogenous color of the graft and the soft consistency indicating good inflow and outflow with no portal hypertension.

The last vascular anastomosis was the hepatic artery anastomosis. The artery used in the anastomosis was the right hepatic, the left hepatic, or the splenic artery according to the placement, length, caliber, compatibility, and flow features of the selected artery. Artery anastomosis was done using microsurgical instruments with monofilament (Prolene 8/0) interrupted sutures.

After finishing the vascular anastomoses, intraoperative Doppler $\mathrm{U} / \mathrm{S}$ was done to assess 
the hepatic artery follow, hepatic vein and portal vein. Then biliary anastomosis was done through duct to duct technique which were more than one anastomosis according to the number of the ducts in the graft, on the other hand hepatico-jejunostomy was considered an option in some cases with multiple small ducts.

Regarding immunosuppression both groups received Certican (Everolimus) and low dose tacrolimus to minimize the incidence of recurrence after transplantation.

\section{Follow up strategy}

The median period of follow up was from 6-48 months. Computed tomography (CT), serum alpha-fetoprotein (AFP) measurements and liver biochemistry were done for all patients at 1 month and 3 months intervals for tumor surveillance then it was done every 6 months along the follow up period. According to the latest American Association for the Study of Liver Diseases guidelines, intrahepatic recurrence was defined as a new lesion by arterial contrast enhancement and portal venous washout.

\section{Statistical methods}

Mean (standard deviation [SD]) or median (interquartile range) were used to express the descriptive statistics. For the univariate comparisons; we used the Fisher's test or Chi square test. As regard the univariate of the survival analysis, Kaplan-Meier method was used to compare the plots created. Significant differences were considered at $\mathrm{P}<0.05$. For all statistical analyses, the CLTR (version 9.2 SAS software), was used.

\section{Results \\ Patient profiles:}

Among the 110 HCC patients, 95 (86.4\%) underwent PLT, while 15 (13.6\%) underwent SLT for $\mathrm{HCC}$ recurrence after the primary resection. In our study a total of 76 PLT recipients were males (80\%), compared with $11(73.3 \%)$ SLT recipients ( $P$ value $=0.012$ ). No significant statistical difference was found regarding the mean age, MELD score and post-transplant microvascular invasion which were (50.6 vs. 52.0$)$, (15.4 vs. 14.6$)$ and ( $82.8 \%$ vs. $86.6 \%$ ) respectively. For patients follow up, there was no significant difference in the median follow up between PLT and SLT recipients, as it was 12.4 months (inter-quartile range between 3.3 28.8 months) and 12.2 months (inter-quartile range between 3 - 29.9 months), respectively.

On the other hand, the pre-operative level of AFP between PLT recipients and SLT recipients showed significant difference as median $134.7 \mathrm{ng} / \mathrm{ml}$, (range between $13.8-1,000 \mathrm{ng} / \mathrm{ml}$ ) vs. median $78.2 \mathrm{ng} /$ $\mathrm{ml}$, (range between $9.1-671 \mathrm{ng} / \mathrm{ml}$ ), respectively. Also, significant difference $(P$ value $=0.001)$ between the 2 groups was observed in the pretransplant treatments which included radiofrequency ablation (RFA), trans-catheter arterial chemo-embolization (TACE), systemic Chemotherapy and in some cases, combination treatments. Patients' characteristics and profiles are summarized in Table $\mathbf{1}$.

\section{Operative characteristics and post-operative complications:}

The operative time was significantly more ( $P$ value $=0.001$ ) in the SLT recipients group, as the median operative time was (10) hours (inter-quartile range between 7- 12 hours) while it was (8) hours (inter-quartile range between 6.5-9.5 hours) in the PLT recipients group.

For the intra-operative loss of blood, the median was $1,700 \mathrm{ml}$ (inter-quartile range between $1,000-3,000 \mathrm{ml}$ ) in PLT recipients compared to 2,000 $\mathrm{ml}$ (interquartile range between $1,200-4,000 \mathrm{ml}$ ) in SLT recipients which was also significant. Although these differences, the length of ICU stay and overall hospital stay after transplantation, were the similar between the 2 groups. Operative characteristics of SLT and PLT recipients are summarized in Table 2.

For all post-operative complications, no statistical significant difference was found between the PLT and SLT recipients. Postoperative complications for all 110 recipients were compared and detailed in Table 3. 
Table 1: Clinical profiles and overall characteristics of patients who underwent PLT and SLDLT for HCC

\begin{tabular}{|c|c|c|c|}
\hline Variable & PLT $n=95$ & SLDLT $n=15$ & \\
\hline Age (years) & $50.62+\backslash-6.61$ & $52.01+/-5.85$ & 0.343 \\
\hline \multicolumn{4}{|l|}{ Gender } \\
\hline Male no. (\%) & $76(80)$ & $11(73.3)$ & NS \\
\hline Preoperative AFP level (median range), ng/ml & $134.72(13.76-1000)$ & $78.23(9.13-670.95)$ & $<0.001$ \\
\hline MELD score & $15.49+-4.87$ & $14.69+-4.22$ & NS \\
\hline \multicolumn{4}{|l|}{ CHILD Pugh classification } \\
\hline A & $17(17.9 \%)$ & $7(46.6 \%)$ & NS \\
\hline B & $32(33.7 \%)$ & $2(13.3 \%)$ & NS \\
\hline C & $46(48.4 \%)$ & $6(40 \%)$ & NS \\
\hline Milan /Extended Milan 9riteria & $95 / 0$ & $15 / 0$ & \\
\hline Number of nodules & $2.05=/-1.39$ & $1.74+/-1.02$ & $<0.001$ \\
\hline Overall size & $4.20+/-1.96$ & $3.64+/-1.31$ & $<0.01$ \\
\hline \multicolumn{4}{|l|}{ Pathological grade } \\
\hline Grade I /complete necrosis & $23(24 \%)$ & $2(13.3 \%)$ & NS \\
\hline Grade II or higher & $72(76 \%)$ & $13(86.6 \%)$ & NS \\
\hline post-transplant micro vascular invasion & $79(82.8 \%)$ & $13(86.6 \%)$ & NS \\
\hline Capsular invasion & $91(96.8 \%)$ & $13(86.6 \%)$ & NS \\
\hline
\end{tabular}

PLT: Primary Liver Transplantation, SLDLT: Salvage Living Donor Liver Transplantation, HCC: Hepatocellular Carcinoma, LDLT: Living Donor Liver Transplantation.

Table 2: Operative data of patients underwent PLT and SLDLT for HCC

\begin{tabular}{lccc}
\hline & PLT & SLDLT & P value \\
\hline Operative time (hours) & $8(6.5-9.5)$ & $10(7-12)$ & $<0.011$ \\
Blood loss (ml) & $1.700(1000-3000)$ & $2000(1200-4000)$ & $<0.011$ \\
ICU stay (days) & $6(5-10)$ & $8(6-15)$ & NS \\
Total hospital stay (days) & $20(15-35)$ & $22(18-37)$ & NS \\
\hline
\end{tabular}

PLT: Primary Liver Transplantation, SLDLT: Salvage Living Donor Liver Transplantation, HCC: Hepatocellular Carcinoma, LDLT: Living Donor Liver Transplantation.

Table 3: Post-operative complications of patients underwent PLT and SLDLT for HCC

\begin{tabular}{lccc}
\hline Post-operative complications & PLT & SLDLT & P value \\
\hline Intra-abdominal collection or abscess & $23(24.2 \%)$ & $3(20 \%)$ & NS \\
Postoperative infection & $27(28.4 \%)$ & $4(26.6 \%)$ & NS \\
Biliary complications & $11(11.6 \%)$ & $2(13.3 \%)$ & NS \\
Renal failure & $3(3.2 \%)$ & $1(6.6 \%)$ & NS \\
Vascular complications & $4(4.2 \%)$ & $1(6.6 \%)$ & NS \\
\hline
\end{tabular}

PLT: Primary Liver Transplantation, SLDLT: Salvage Living Donor Liver Transplantation, HCC: Hepatocellular Carcinoma, LDLT: Living Donor Liver Transplantation.

\section{Survival analysis}

All patients were selected according to Milan criteria. The 1 year, 3 year and 5 year over-all survival and disease-free survival rates were analyzed in the SLT and PLT selected patients.
Similar rates were observed as regard the overall survival between the SLT and PLT recipients as the 1 year, 3 year, and 5 year overall survival rates were $94.5 \%, 87.7 \%$, and $84.9 \%$ in PLT recipients versus $93.3 \%, 86.7 \%$, and $86.7 \%$ in SLT recipients (P 0.868) (Figure 1). 


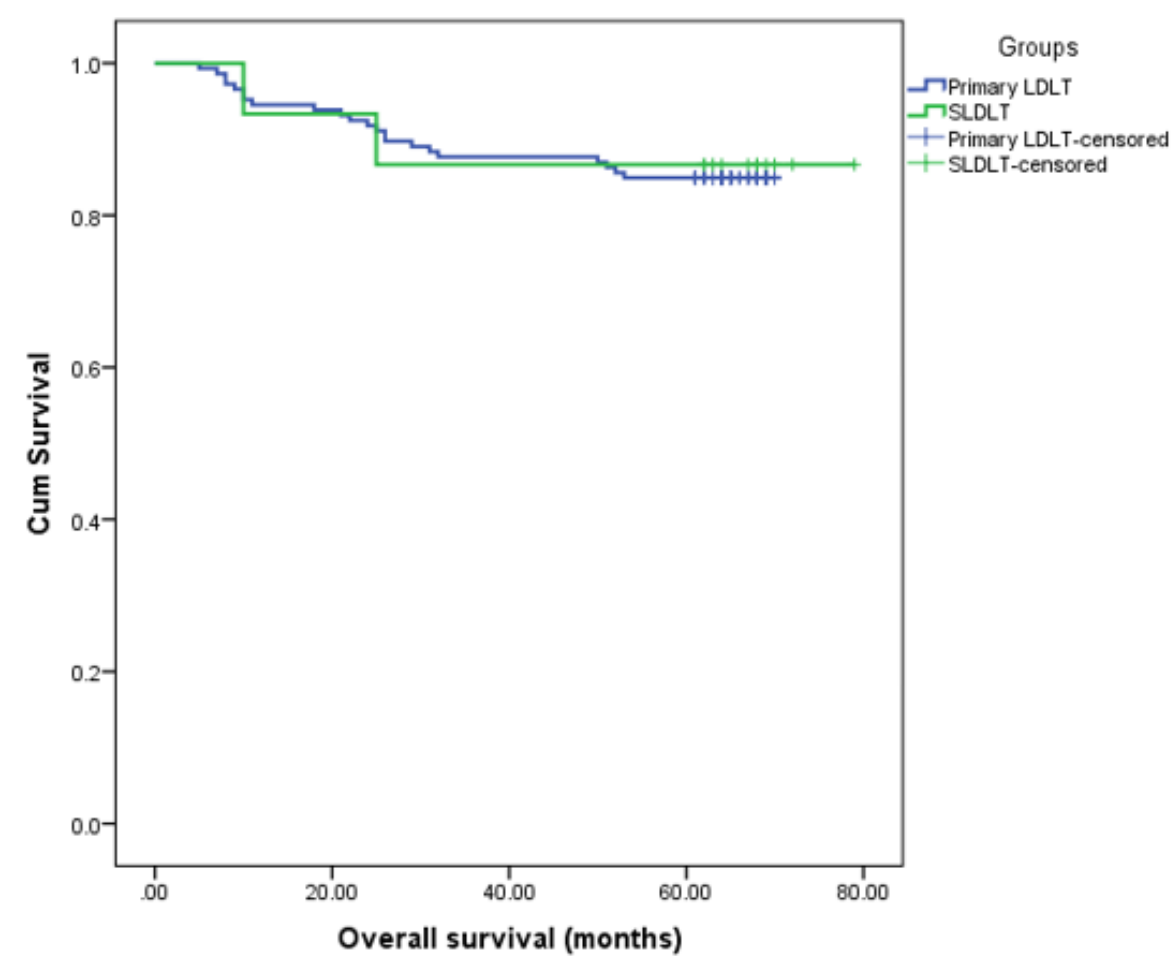

Fig 1: Kaplan Mayer Analysis for overall survival at 1, 3 and 5 years for the two studied groups.

However, the 1 year, 3 year and 5 year disease-free survival rates were $94.5 \%, 93.0 \%$, and $92.1 \%$ in PLT recipients, compared to recurrence occurred in 1 patient after 9 months $(6.7 \%)$ in SLT recipients which was not significant difference statistically between both groups with $P$ value $=0.907$ (Figure 2).

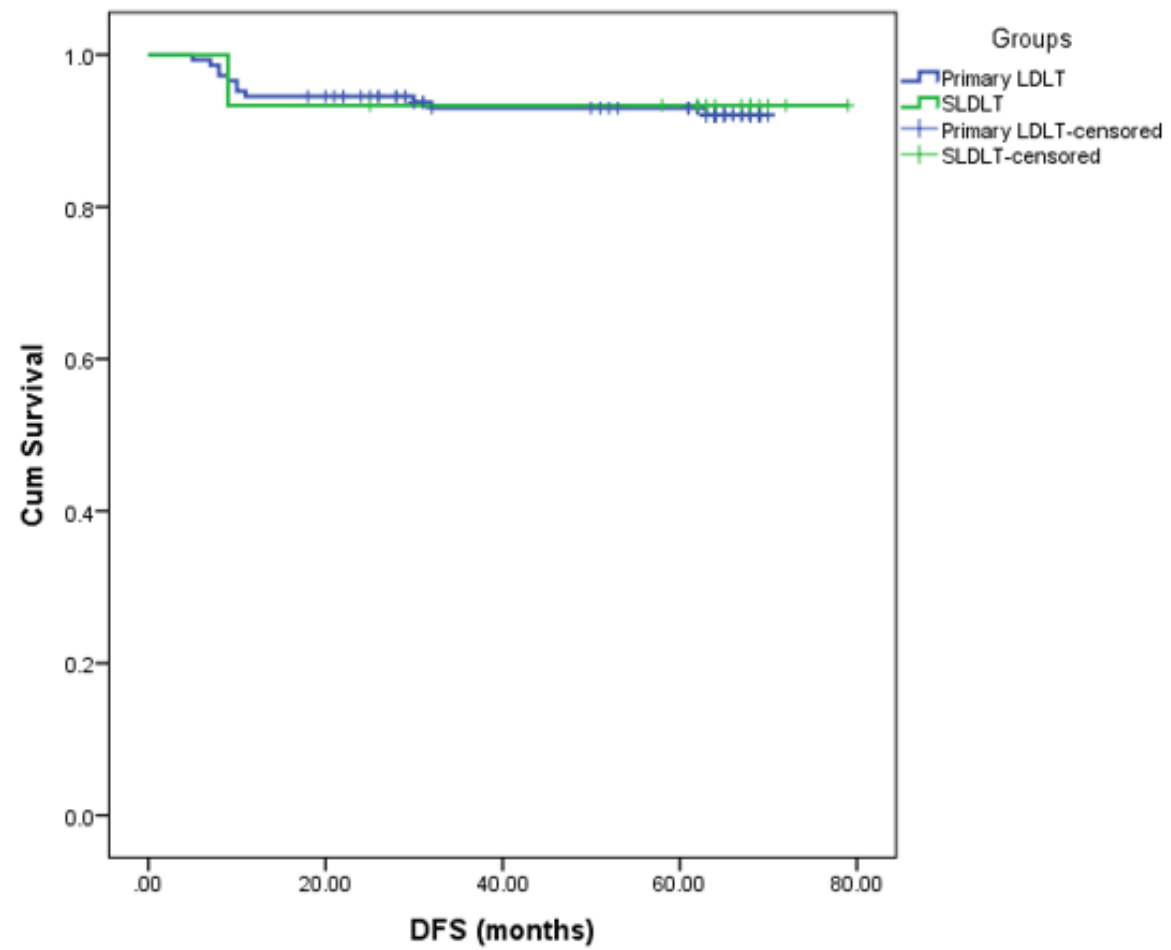

Fig 2: Kaplan Mayer Analysis for disease free survival at 1, 3 and 5 years for the two studied groups. 


\section{Discussion}

Our study showed that SLT recipients and PLT recipients were similar as regard the over-all survival and disease-free survival.

As liver transplantation has been rapidly developed recently, it is now considered the ideal treatment for HCC patients as a curement option for both the tumor and the underlying liver disease. Mazzaferro et al. reported that patients within Milan criteria that underwent liver transplant showed 4-year overall survival and recurrence free survival rates of $85 \%$ and $92 \%$ respectively. ${ }^{8}$ In our study the recipients within Milan criteria had a survival rate of $84.3 \%$ over 5 years.

As a result of the donor organ shortage which became a worldwide limitation, the long term waiting lists may lead to tumor progression and dropout from the waiting list as a consequence. ${ }^{11}$ Yao and his colleagues' study showed that incidence of dropout from the waiting list is increased with longer waiting period, as it was $7.2 \%$ with 6 months, $37.8 \%$ with 12 months, and $55.1 \%$ with 18 months. ${ }^{12}$ It was reported by Del Gaudio et al. that 5 year overall survival rate for liver resection was higher than that for liver transplantation, at $66 \%$ and $58 \%$ respectively ${ }^{13}$ On the other hand Majno et al. identified 4 main parameters affecting the SLT recipients' outcome with life expectancy of 7.8 years compared to 8.8 years for PLT recipients. ${ }^{14}$ As a result, primary liver resection may play a role to prevent tumor advancement till a compatible donor is available as then SLT will be an option if HCC recurs.

Although, many controversies have been about SLT. In our study, 95 of 110 HCC patients (86.4\%) underwent PLT and 15 patients (13.6\%) underwent SLT for HCC recurrence following primary resection. Number of patients underwent PLT were more than SLT due to the fact of that PLT offers higher quality of life with satisfactory survival, whilst little is known about the SLT outcomes. Belghiti et al. indicated the similar rates of 3-year and 5-year over-all survival in PLT and SLT patients ( $82 \%$ vs. $82 \%$ and $59 \%$ vs. $61 \%){ }^{15}$ Also same findings were found in Del Gaudio et al. ${ }^{13}$ and Vennarecci et al. studies. ${ }^{16}$ On the other hand, Adam and his colleagues reported a higher recurrence risk ( $54 \%$ vs. $18 \%$ ), with less 5 -year over-all survival and disease-free survival in SLT recipients than PLT recipients of ( $41 \%$ vs. $61 \%)$ and $(29 \%$ vs. $58 \%)$, respectively. ${ }^{17}$

Our analysis showed no significant difference as regard the 1 year, 3 year, and 5 year overall survival rates between SLT and PLT patients. However, differences were significantly found in the pretransplant status as the median tumors number, largest tumor diameter and pre-operative AFP level. This was may be due to in SLT recipients who had strict follow up after the primary liver resection and prior to transplantation to early detect any recurrence.

In our results we found that the PLT and SLT recipients had similar overall survival rates, inspite of the pretransplant status differences.

In fact, primary resection is considered very beneficial for the patients with early HCC, especially peripheral lesions, to keep them alive without recurrence. Close follow up following the primary resection is mandatory to detect any recurrences, also both SLT and PLT results are similar. ${ }^{15}$ However, for patients with inadequate liver reserve, PLT is the best option of treatment. ${ }^{18}$

As regard other factors like surgical difficulty for SLT recipients due to the presence of adhesions of the previous liver resection, we observed that the intraoperative loss of blood and the time of operation were more in the SLT group. However, the total hospital stay or ICU stay was similar after transplantation with no significant difference which indicated that SLT and PLT recipients have the same recovery. It was also documented that the time of operation, intraoperative loss of blood, post operative ICU stay, and in-patient total hospital stay were the same between SLT and PLT recipients by Vennarecci et al. ${ }^{16}$ and Belghiti et al. ${ }^{15}$ Moreover, we believe that with the improvement of the learning curve and more experience accumulation with more cases of SLT, the operative time and intraoperative loss of blood will be much more controlled.

Our study also showed that the surgical procedure related post-operative complications did not increase in the SLT recipients, which indicates the technical feasibility of the procedure in comparison to PLT. Recently laparoscopic liver resections were found to facilitate the liver transplant operation in comparison to open resections as it reduces the time of operation, loss of blood, and requirement of transfusion with the conclusion of preferred laparoscopic liver resections to open ones in potential transplant candidates, when feasible. ${ }^{11}$

Great opportunity is offered by LDLT to supply the transplantable organs, therefor it can eliminate the prolonged waiting period and risk of dropout. Also, it was reported that patients with HCC would benefit more from LDLT rather than waiting for a cadaveric organ more than 7 months as regard life expectancy and cost-effectiveness. ${ }^{19}$

Hwang et al. noted that the 1 year, 3 year, and 5 year overall survival rates were $80 \%, 80 \%$, and 
$80 \%$ for the salvage group of LDLT for patients within Milan criteria, however, it was $87.8 \%, 80.1 \%$, and $74.8 \%$ for the primary LDLT. ${ }^{20}$ In our study, we found no significant difference between SLT and PLT recipients with LDLT as regard the overall survival and disease-free survival rates, although the higher risk of recurrence in the SLT group.

Due to our retrospective study, we had some restrictions as regard the observational data analysis, related to the quality and depth of the data available. Being not randomly assigned study, unmeasured patient characteristics to confound the results is potentially present. No doubt that the gold standard for clinical studies is still the prospective, randomized, multicentre trials, which we cannot perform such analysis unfortunately.

Inadequate information about the non-transplant therapies, as secondary resection or RFA after recurrence to compare it with liver transplant after recurrence as in SLT recipients, is considered also another limitation. Therefore, possible differences in prognosis after these treatments cannot be assessed. It was shown in previous studies that the overall survival for PLT was superior to nontransplant therapies. In our study we found that the survival was the same between PLT and SLT patients. As a result we may conclude that the survival rate of SLT patients would be higher than non-transplant therapies.

Inspite of all these restrictions, when we compare our study to those previously published, we found that our assessment of SLT recipients is more comprehensive. Also in future, assessing the postrecurrence treatments will help us to find whether these will affect the patients' survival after primary resection, so as to provide a better guidance for making a decision that improves the outcomes.

As a conclusion, our study shows that SLT is a safe and technically feasible procedure for patients that developed HCC recurrence following primary liver resection. Also, the survival rates were similar to PLT, which makes SLT considered as the preferable choice of treatment for HCC recurrence patients. Due to shortage of deceased donor organs, salvage LDLT might be accepted as a good alternative option.

\section{References}

1. Bosch FX, Ribes J, Diaz M, Cleries R: Primary liver cancer: Worldwide incidence and trends. Gastroenterology. 2004; 127: S5-S16.

2. Sangiovanni A, Del Ninno $E$, Fasani $P$, et al: Increased survival of cirrhotic patients with hepatocellular carcinoma detected during surveillance. Gastroenterology. 2004; 126: 1005-1014.

3. Poon RT, Fan $\mathrm{ST}$, Lo CM, Ng IO, Liu CL, et al: Improving survival results after resection of hepatocellular carcinoma: A prospective study of 377 patients over 10 years. Ann Surg. 2001; 234: 63-70.

4. Ercolani G, Grazi GL, Ravaioli M, Del Gaudio M, Gardini A, et al: Liver resection for hepatocellular carcinoma on cirrhosis: Univariate and multivariate analysis of risk factors for intrahepatic recurrence. Ann Surg. 2003; 237: 536-543.

5. Pugh RN, Murray-Lyon IM, Dawson JL, Pietroni MC, Williams R: Transection of the oesophagus for bleeding oesophageal varices. Br J Surg. 1973; 60: 646-649.

6. Pugh RN, Murray-Lyon IM, Dawson JL, Pietroni MC, Williams R: Transection of the oesophagus for bleeding oesophageal varices. Br J Surg. 1973; 60: 646-649.

7. Poon $\mathrm{RT}$, Fan $\mathrm{ST}$, Lo $\mathrm{CM}$, Liu $\mathrm{CL}$, Wong J: Long-term survival and pattern of recurrence after resection of small hepatocellular carcinoma in patients with preserved liver function: Implications for a strategy of salvage transplantation. Ann Surg. 2002; 235: 373-382.

8. Mazzaferro V, Regalia E, Doci R, Andreola S, Pulvirenti $A$, et al: Liver transplantation for the treatment of small hepatocellular carcinomas in patients with cirrhosis. $\mathbf{N}$ Engl $\mathbf{J}$ Med. 1996; 334: 693-699.

9. Bismuth $\mathrm{H}$, Majno $\mathrm{PE}$, Adam R: Liver transplantation for hepatocellular carcinoma. Semin Liver Dis. 1999; 19: 311-322.

10. Strong RW: Transplantation for liver and biliary cancer. Semin Surg Oncol. 2000; 19: 189-199.

11. Laurent A, Tayar C, Andreoletti M, Lauzet JY, Merle JC, et al: Laparoscopic liver resection facilitates salvage liver transplantation for hepatocellular carcinoma. $\boldsymbol{J}$ Hepatobiliary Pancreat Surg. 2009; 16: 310-314.

12. Yao FY, Bass NM, Nikolai B, Merriman R, Davern TJ, et al: A follow-up analysis of the pattern and predictors of dropout from the waiting list for liver transplantation in patients with hepatocellular carcinoma: implications for the current organ allocation policy. Liver Transpl. 2003; 9: 684-692. 
13. Del Gaudio M, Ercolani G, Ravaioli M, Cescon M, Lauro $A$, et al: Liver transplantation for recurrent hepatocellular carcinoma on cirrhosis after liver resection: University of Bologna experience. $\mathbf{A m}$ J Transplant. 2008; 8: 1177-1185.

14. Majno PE, Sarasin FP, Mentha G, Hadengue A: Primary liver resection and salvage transplantation or primary liver transplantation in patients with single, small hepatocellular carcinoma and preserved liver function: An outcomeoriented decision analysis. Hepatology 2000; 31: 899-906.

15. Belghiti J, Durand F: Hepatectomy vs. liver transplantation: A combination rather than an opposition. Liver Transpl. 2007; 13: 636-638.

16. Vennarecci G, Ettorre GM, Antonini M, Santoro $R$, Maritti $M$, et al: First-line liver resection and salvage liver transplantation are increasing therapeutic strategies for patients with hepatocellular carcinoma and child a cirrhosis. Transplant Proc. 2007; 39: 1857-1860.
17. Adam R, Azoulay D, Castaing D, Eshkenazy R, Pascal $G$, et al: Liver resection as a bridge to transplantation for hepatocellular carcinoma on cirrhosis: a reasonable strategy? Ann Surg. 2003; 238: 508-518; discussion 518-509.

18. Facciuto ME, Koneru B, Rocca JP, Wolf DC, Kim-Schluger $L$, et al: Surgical treatment of hepatocellular carcinoma beyond Milan criteria. Results of liver resection, salvagetransplantation, and primary liver transplantation. Ann Surg Oncol. 2008; 15: 1383-1391.

19. Sarasin FP, Majno PE, Llovet JM, Bruix J, Mentha $\mathrm{G}$, et al: Living donor liver transplantation for early hepatocellular carcinoma: A lifeexpectancy and cost-effectiveness perspective. Hepatology. 2001; 33: 1073-1079.

20. Hwang S, Lee SG, Moon DB, Ahn CS, Kim KH, et al: Salvage living donor liver transplantation after prior liver resection for hepatocellular carcinoma. Liver Transpl. 2007; 13: 741-746. 